\title{
Observing with the VLT Interferometer
}

\author{
M. Wittkowski ${ }^{a}$, P. Ballester ${ }^{a}$, T. Canavan ${ }^{a}$, F. Comerón ${ }^{a}$, C.A. Hummel ${ }^{b}$, A. Kaufer ${ }^{b}$, \\ S. Marteau ${ }^{a}$, G. Mathys ${ }^{b}$, S. Morel $^{b}$, P. Naß ${ }^{a}$, I. Percheron ${ }^{a}$, M. Peron ${ }^{a}$, M. Petr-Gotzens $^{a}$, \\ F. Rantakyrö ${ }^{b}$, A. Richichi ${ }^{a}$, M. Schöller ${ }^{b}$, D. Silva ${ }^{a}$, M. van den Ancker ${ }^{a}$, and A. Wallander ${ }^{a}$ \\ ${ }^{a}$ European Southern Observatory, Karl-Schwarzschild-Str. 2, D-85748 Garching, Germany \\ ${ }^{b}$ European Southern Observatory, Casilla 19001, Santiago 19, Chile
}

\begin{abstract}
The ESO Very Large Telescope Interferometer (VLTI) is the first general-user interferometer that offers nearand mid-infrared long-baseline interferometric observations in service mode as well as visitor mode to the whole community. Regular VLTI observations with the first scientific instrument, the mid-infrared instrument MIDI, have started in ESO observing period 73, for observations between April and September 2004. Regular observations with the near-infrared instrument AMBER are planned to follow soon after. The efficient use of the VLTI as a general-user facility implies the need for a well-defined operations scheme. The VLTI scheme follows the established general operations scheme of the other VLT instruments. Here, we present from the users' point of view the VLTI specific aspects of this scheme beginning from the preparation of the proposal until the delivery of the data.
\end{abstract}

Keywords: long-baseline interferometry, VLTI, operation, observing

\section{INTRODUCTION}

The ESO VLT (Very Large Telescope) Interferometer, located on Cerro Paranal in northern Chile, is now being offered as a general user interferometer to the whole astronomical community in service mode as well as in visitor mode. This facility will ultimately (first generation instrumentation) comprise the four fixed 8 m diameter VLT Unit Telescopes (UTs), four $1.8 \mathrm{~m}$ diameter Auxiliary Telescopes (ATs) which can be moved over an array of 30 stations, the scientific instruments AMBER (three-beam instrument) and MIDI (two-beam instrument) for observations at near-infrared and mid-infrared wavelengths respectively, the fringe tracker FINITO, and a dualfeed facility (PRIMA) for astrometry and phase referencing. At present, the VLTI is offered with the MIDI instrument and a small number of UT baselines. The addition of the AMBER instrument and the first two ATs is expected for 2005. The use as a general-user facility, in particular with service mode observations, implies the need for a well-defined and efficient operations scheme. Service mode observing has been perceived by ESO since the early days of the planning of VLT operations as a key component in optimizing the scientific return and the operational efficiency (e.g. Comerón et al. ${ }^{4}$ ). Regular science operations including service mode observations started at the ESO VLT in 1999 with the first single VLT/UT (ANTU) and the instruments ISAAC and FORS1 (e.g. Comerón et al. ${ }^{4}$ ). The VLTI operations scheme profits enormously from this experience and the established infrastructure. It follows the general VLT operations scheme from the initial preparation of the proposal until the delivery of the data. The same level of support is offered to users of the VLTI as to users of any other instrument of the single VLT/UTs. By now, one complete cycle of VLTI operations with the MIDI instrument has been gone through, from the initial preparation of the proposals, first observations in service mode and visitor mode, until the completion of the first runs and their data packaging. Here, we present an overview on the VLTI-specific aspects of the complete VLT operations scheme. We focus on the users' point of view, and include operational aspects which are not influenced by the user but interesting for the understanding of the scheme.

While this article provides an overview on the complete VLTI observing scheme, several separated aspects are described in more detail in other presentations of this conference. Glindemann et al. ${ }^{6}$ describe the technical advances of the VLTI, Morel et al. ${ }^{11}$ describe the preparation of the first scientific instrument, MIDI, for operations, Rantakyrö et al. ${ }^{15}$ discuss the organization of the VLTI observations on Paranal, Percheron et al. ${ }^{14}$ focus

Send correspondence to M. Wittkowski, E-mail: mwittkow@eso.org 
on the MIDI quality control and instrument trending aspects. Galliano et al. ${ }^{5}$ discuss the selection of optimum subsets of available array configurations with four ATs and 30 possible stations for each of them. Aspects on the selection of calibration stars have previously been presented by Percheron et al. ${ }^{12,13}$ The data flow system, including the pipeline data reduction for present and future VLTI instrumentation is described by Ballester et al. ${ }^{3}$ Technical details of VLTI observation planning tools are discussed by McKay et al. ${ }^{8}$ The general ESO telescope time allocation process is presented by Alves \& Breysacher. ${ }^{1}$ Scientific prospects of the MIDI and AMBER instruments are presented by Leinert ${ }^{7}$ and Malbet et al., ${ }^{10}$ respectively. An overview of scientific results, mainly obtained during VLTI commissioning, can also be found in Richichi \& Paresce. ${ }^{16}$

Here, we introduce the main characteristics of the general VLT operations scheme in Sect. 2 and the specific requirements that arise for interferometry in Sect. 3. In Sect. 4 we describe the VLTI specific aspects of the whole VLTI operation scheme. In Sect. 5 we introduce the preparation tools that are available for VLTI observations. Finally, in Sect. 6 we discuss the experience from past and present VLTI observing periods and provide an outlook to the upcoming observing periods.

This presentation is, if not explicitly stated otherwise, based on the current status of the VLTI operation scheme, i.e. in ESO observing period P74, for observations between October 2004 and March 2005. The scheme is of course evolving with more experience and with the additions of further instrumentation.

\section{THE GENERAL VLT OPERATIONS SCHEME}

A recent description of the ESO VLT operations scheme can be found in Comerón et al. ${ }^{4}$ The ESO VLT scheme allows astronomers to submit visitor mode or service mode observation programmes. In visitor mode, the astronomer is present at the telescope and can adopt the observation programme to specific target properties, changing observation conditions, or calibration needs. In service mode, the observation details and constraints are submitted to ESO by the requesting astronomer beforehand, and the observations are scheduled by a flexible queue and carried out by ESO staff. The data are processed and sent to the requesting astronomer.

The service mode scheme can be divided into different phases. At phase 1 proposal preparation the scientific proposal is prepared and submitted electronically to ESO. Information on the details of the offered instruments and observing modes can be found in the Call for Proposals (CfP) which is released twice a year, around the first of March and the first of September. The Visiting Astronomers Section supports the proposal submission and time allocation processes (www.eso.org/observing/proposals), and the User Support group (USG, www.eso.org/org/dmd/usg/index.html) questions on instruments, their possibilities, and strategies. Technical information on the instruments is available at www.eso.org/instruments. The deadlines for proposal submission are typically 1 October and 1 April. Afterwards, the proposals are evaluated by the Observing Programmes Committee (OPC), and a long-term schedule is created which combines the visitor mode runs and service mode periods in the best possible way. The allocation of the observing programmes in the long-term schedule is done in strict accordance with the OPC scientific ratings, and takes also into account the technical feasibility, target distribution on sky, and user-specified constraints on the execution conditions (see Silva ${ }^{17}$ ). Once the long-term schedule is ready and the list of scheduled runs is finalized, the Principal Investigators are informed about the time allocated to each of their runs. This marks the beginning of the phase 2 proposal preparation. Here, the users provide the set of Observation Blocks (OBs) which fully define their observations, including the user constraints on observation conditions, which are prepared with the Phase 2 Preparation Program (P2PP*). Each single OB specifies an individual observing sequence that is scheduled and executed without interruption and should usually not take more than 1 hour of execution time, in order to retain scheduling flexibility and optimizing the observations under varying atmospheric conditions. One observing run consists typically of a number of OBs which are scheduled and executed independently. Although some types of programmes can produce scientific results with partial datasets, ESO gives high priority to completing all the observations composing a given run rather than partially executing as many runs as possible. Detailed information about this phase including general and instrument-specific instructions can be found at www.eso.org/org/dmd/usg/ServiceMode.html. The User Support Group provides assistance with problems and answers any questions that arise during this phase. The phase 2 packages, consisting of OBs and a set of user-provided auxiliary information and execution

*www. eso.org/observing/p2pp 
instruction items, are also reviewed by the User Support Group for possible optimization, for compliance with policies, technical correctness, and consistency with the phase 1 information as approved by the OPC. Once certified the runs are included in the medium term schedule queues that are provided daily to Paranal science operations. The short term scheduling, i.e. the actual sequence of observations carried out during a given night, is prepared by the astronomer in charge for service mode observations on Paranal. The observations are checked for compliance with the user-defined constraints right after their execution. Once an observing run is completed, a data package is prepared and sent to the PI. This data package contains all the science data associated with the completed run, the corresponding calibration data, and a variety of data products, when a data processing pipeline is available.

The visitor mode scheme is the same until the announcement of the observing time. The tools used, including $\mathrm{P} 2 \mathrm{PP}$ and the observation preparation tools, are common to both service and visitor observations. Visiting astronomers can prepare their observations previous to their arrival on the mountain. The point of contact during the preparation of the observing material is the Paranal Science Operations group, instead of the User Support Group in Garching as for service mode. Information about the visitor mode scheme is available at www . eso.org/observing/p2pp/VisitorMode.html.

\section{SPECIFIC REQUIREMENTS FOR INTERFEROMETRY}

The most important additional implications for the operations scheme that result from interferometric observations arise from three requirements: (1) the need for frequent calibration of the interferometric transfer function, i.e. the calibration of atmospheric and instrumental visibility losses, (2) the need for observation sequences that make use of the earth rotation to realize different projected baseline lengths and azimuth angles, and (3) the use of different array/baseline configurations. All of these requirements have an impact on the long-term, medium-term, and short-term scheduling of the observations. Additional difficulties during the preparation of the interferometric observations may arise from the sometimes a priori unknown source structure, and hence the unknown correlated magnitude and feasibility of the sources.

Calibration. In order to obtain a sufficient accuracy of the interferometric results, the evolution of the atmospheric and instrumental transfer function has to be monitored frequently during each night. In order to reach this, observing sequences with alternating observations of scientific targets and calibration stars are needed. At the moment, a user is requested to submit a calibration star OB for each science star OB. This pair of OBs is then executed sequentially without interruption. The two OBs of the pair are only considered completed if both OBs of the pair were successfully executed. At the moment, only such pairs of OBs are accepted. Different pairs of science target/calibration star OBs are executed independently. In the course of the night, this leads to an alternating observing sequence of science star OBs and calibration star OBs. The data taken on all calibration stars are public once they arrive in the archive. Hence, each PI can make use of the information from all calibration stars observed in the respective night. The association between the science target and the calibration star $\mathrm{OB}$ is reached by inserting the name of the corresponding calibration star OB in the science star $\mathrm{OB}$, and this information is used by the astronomer on Paranal in charge of the service mode observations. For future development, the linking between science star and calibration star observations could be done in a more automatic way, and more complex observation sequences could be accommodated (provided that they do not take more time than about the maximum uninterrupted execution time of 1 hour). In visitor mode, any sequence of science target and calibration star OBs can be chosen by the visiting astronomer.

To obtain a good accuracy for the calibrated visibility measurements, it is important to establish a list of calibration stars which are suitable, i.e. for instance which do not show strong spectral features, are not variable or multiple sources. The exact influence of calibrator characteristics, such as distance on sky and brightness, on the accuracy of the final result have not yet been fully investigated for VLTI observations. Usually, the selection is based on a reasonable compromise between different characteristics such as brightness (not much fainter than the target), distance on sky (close to the target), diameter (unresolved or at least clearly smaller than the target), and maybe spectral type or other characteristics which are important for the respective observing mode. For this search of suitable calibration stars two concepts have been discussed. The first concept is based on searches in large catalogs like CDS in Strasbourg, France (cdsweb.u-strasbg.fr), based on specified criteria. The second 
concept includes the creation of a smaller set of calibration stars based on known objects in the literature. If necessary, complementary measurements can be taken (see e.g. Kimeswenger et al. ${ }^{9}$ ). This strategy reduces the number of calibrators in the list, but it preserves objects which have already been studied. Furthermore, with a smaller list, the frequency of observations will be greater, and hence detailed knowledge of the sources will be acquired more rapidly. For the current MIDI observations, a calibration selection tool (CalVin, see below) is offered that allows the user to select a calibrator from a set of currently about 200 stars. This list has been established by the MIDI consortium, based on model atmosphere fits to obtained photometric data. ESO recommends the use of this tool.

Observation sequences. The scientific goal of an interferometric observation campaign can often only be reached if visibility measurements at different points in the $u v$-plane, i.e. measurements using different values of $\lambda / B$ ( $\lambda$ being the wavelength of observations and $B$ the projected baseline) and different projected azimuth angles, are combined. The realization of different $u v$-points can be reached by combining different ground baselines and/or by making use of the earth rotation for a given ground baseline. It may also be reached by using a range of wavelengths, as offered by the MIDI and AMBER instruments, but this requires assumptions on the source structure as a function of wavelength. For VLTI observations, each instantaneous visibility measurement (with 2 telescopes for MIDI, 2 or 3 telescopes for AMBER, and the chosen wavelength dispersion) requires the submission of one OB. Multiple observations of the same source at different times or with different baseline configurations require the submission of multiple OBs. For service mode operations, these OBs are executed independently. The projected baseline length, the projected azimuth angle, and the zenith distance are defined by a given hour angle or local sidereal time (LST) at which the observation is executed. The VLTI-specific constraints (in particular hour angle) can be described as a part of the OB so that OBs can be effectively considered as stand-alone entities and support for linked observations is generally not required. In order to allow the user to plan observations which combine measurements at different $u v$-points, the following constraints can be inserted in each individual OB: (1) the array configuration, (2) an interval of the local sidereal time during which the observation is executed. In addition, date intervals can also be given in order to allow, for instance, the monitoring of variable source. Furthermore it can be requested that all OBs of one run are executed within a certain time range, which may be needed for variable targets as well. The preparation and planning of these observation sequences is supported by the visibility calculator VisCalc (see below), which calculates visibility values for a model source as a function of hour angle and baseline configuration. For some VLTI observations, maybe in particular for imaging campaigns with AMBER, it will be mandatory that (almost) all OBs of a run are executed in order to reach the scientific goal (the reconstruction of an image). This need is supported by the general VLT rule that a high priority is given to completing all the observations composing a given run rather than partially executing as many runs as possible (see Sect. 2).

Scheduling of VLTI observations. The baseline choice for a particular visibility measurement which is selected by the user, is inserted in the OB like a constraint on the observation condition such as seeing or sky transparency. However for the scheduling of the observation, there is a major difference between this observational constraint on the baseline choice as compared to constraints on the atmospheric conditions: the array/baseline configuration is scheduled blockwise beforehand for the whole observing period, while the weather conditions during each particular night are not predictable. The user's choice for the intervals of the Local Sidereal Time at which the observation has to be carried out is known as well at the beginning of each observing period. This addition of baseline choice and intervals of Local Sidereal Time complicate the long-term scheduling, the medium-term scheduling, as well as the short-term scheduling. First, the baseline configurations are scheduled block-wise for the whole observing period. At this point, it is unknown how much actual observing time will be available for each baseline after the subtraction of downtimes due to weather, technical reasons, etc. While for single VLT observation these unknowns average for all service mode programmes of the whole semester, they average for VLTI observations only for each specific baseline configuration. Second, while multiple OBs of each source for regular single VLT observations can be carried out at any time regardless of hour angle as long as the target is below a certain user-specified airmass, multiple OBs of the same source for VLTI observations usually require a range of specified hour angle values. Finally, it is of course more complex for the short-term scheduling 


\begin{tabular}{ll} 
Parameter & Value choices \\
\hline For each run: & \\
Telescope & VLTI \\
Instrument & MIDI \\
Constraints on seeing, moon, transparency & any \\
Obs. mode & service mode or visitor mode \\
Instr. mode & PRISM $(\lambda / \Delta \lambda \sim 30)$ or GRISM $(\lambda / \Delta \lambda \sim 230)$ \\
For each target and instrument mode: & \\
\hline V magnitude of the target & $<16$ for the guiding performance \\
$\begin{array}{l}\text { Requested Spectral Resolution } \lambda / \Delta \lambda \\
\text { Magnitude at wavelength of observations }\end{array}$ & 30 or 230 \\
Central wavelength of observation & $10.3 \mu \mathrm{m}$ \\
$\begin{array}{l}\text { Size of the target at wavelength of observation } \\
\text { Baseline }\end{array}$ & 'UT2-UT3-47m', 'UT3-UT4-62m', 'UT2-UT4-89m' \\
$\begin{array}{l}\text { Expected visibility } \\
\text { Correlated flux (flux } \times \text { visibility) }\end{array}$ & $>2$ Jy for PRISM, $>1$ Jy for PRISM in visitor mode, \\
& $>10$ Jy for GRISM.
\end{tabular}

Table 1. MIDI-specific parameters in the ESO application form for observing time (P74 version, for observations between October 2004 and March 2005).

during the night to accommodate the additionally required LST interval, while being limited to only those OBs which require the currently available baseline configuration. As a result, it is advisable not to require stringent constraints on the atmospheric conditions in order not to decrease dramatically the likelihood that all constraints will be met at some time during the observing period.

\section{THE VLTI SPECIFIC ASPECTS OF THE OPERATIONS SCHEME}

Here we describe the most important interferometry-related aspects that have to be considered for the different phases of the operations scheme, based on the requirements in Sect. 3.

Preparation of the proposal (Phase 1). For the planning of the observation, the visibility calculator VisCalc is available (see below). The offered instrument modes are listed in the CfP, and the instrument details are documented in the instrument manual (see www.eso.org/instruments). In the proposal text, the requested baseline configurations, the instrument modes and the expected visibility values have to be specified. Table 4 gives an overview on the VLTI-specific parameters in the ESO proposal form. Currently, the exact LST intervals and the calibrator information is not required for the phase 1 proposal preparation, but is part of the phase 2 preparation.

Preparation of the observing material (Phase 2). The standard VLT entries specifying the target information, instrument parameters, and the constraint set on the atmospheric conditions are entered at this stage. In addition, for each OB the requested LST interval can be specified, as well as the baseline choice. Figure 1 shows an example of the phase 2 preparation tool (P2PP) illustrating how the baseline configuration and LST interval are inserted. Special requirements and a finding chart at optical wavelengths (for identifying the target at the VLTI Coudé camera) is provided as well at this stage.

Operations phase. During operations, the scheduling tool (called "OT") can select only the OBs for the actual baseline configuration. It can also select OBs for specified LST ranges. A night-log is produced that gives detailed information on the observational parameters of the executed OBs. In particular, it is checked that the user-specified constraints on the observing conditions are met, like for the VLT instrument. In addition 


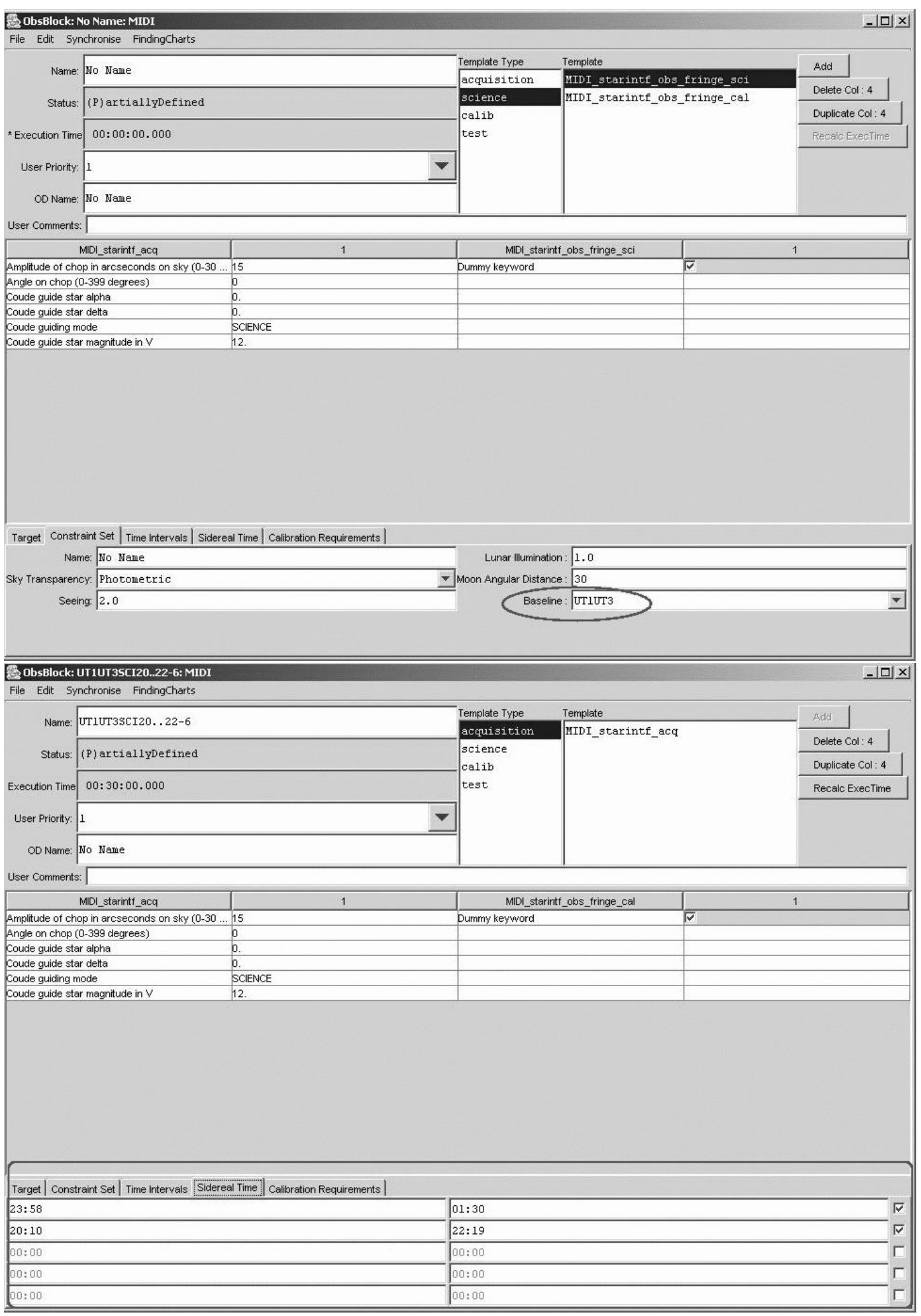

Figure 1. Sample pages of the phase 2 preparation tool (p2pp) illustrating how the information on the baseline choice (top) and LST interval (bottom) is inserted (status of P74). 
it is checked that the requested baseline configuration was used, that the LST interval was met, and that interferometric fringes were detected by the system. For VLTI operations, a lot of subsystems have to function smoothly together. This includes the telescopes, the guiding systems at the Coudé foci (tip-tilt or adaptive optics), the delay line systems together with the variable curvature mirrors, the instrument itself, and ultimately the external fringe tracker FINITO. The detection of fringes proofs that all systems work and is therefore a very important quality control parameter. However, quality control of all separate subsystems is important as well, and the corresponding quality control parameters that are assessed during the observations are being defined. An automatic data-reduction pipeline reduces the data right after the execution of the OB, and produces additional information on their quality. For more information about this phase, see Rantakyrö et al. ${ }^{15}$

Quality control and data delivery. Once the data arrive in the ESO archive in Garching, the quality of the pipeline-processed data is assessed in more detail (see Percheron et al ${ }^{14}$ ). The data package is sent to the Principal Investigator of the programme once a run is completed. This package contains all the raw data associated to the programme (e.g., acquisition images on both telescopes, fringe tracking data, photometric calibrations), and the technical calibration data which are used to calibrate the science data. The data on the calibration star that was requested by the PI is included in this package. The obtained instrumental transfer function for the respective night, as obtained by the pipeline-processed data of the calibration stars, is part of the data package as well. The raw data from all calibration stars obtained during each night are public and can be requested from the ESO archive.

\section{PREPARATION TOOLS FOR VLTI}

User documentation of VLTI and the instruments. For the preparation of VLTI observations, documentation on the instruments (www.eso.org/instruments), as well as on the proposal preparation process (www.eso.org/observing) is available. The preparation of observations is supported by the User Support Group (www.eso.org/org/dmd/usg/index.html).

The webtools VisCalc and CalVin In order to plan an interferometric observation and to assess its feasibility, one needs adequate tools to model the visibility for a specified array configuration, taking into account constraints like shadowing effects or the range of the delay lines. In addition, appropriate calibration stars must be selected. Two specific tools are provided for this purpose: the VLTI Visibility Calculator (VisCalc) and the calibrator selection tool (CalVin). VisCalc provides calculations of simulated dispersed visibilities based on software models of the VLTI instruments. The declination and spectral energy distribution, as well as the source geometry, are parameters used to specify the observation target. Visibilities are calculated analytically for uniform disks, Gaussian disks and binaries. Visibilities may also be calculated numerically for a user-provided brightness distribution which is uploaded as a FITS file. The user-specified observation conditions include the starting hour angle and the duration of the observation, as well as the instrument and array configuration. Different results can be displayed (Fig. 2) including the $u v$-tracks, the input image and its Fourier transform, plots of visibility versus time, visibility squared versus time, loss of correlated magnitude, or the illumination distribution.

The calibrator selection tool (CalVin) provides a similar interface and involves a two stage selection process. On the first input page, the target coordinates, the array and instrument configurations can be selected. The default search criteria are displayed on an intermediate page which allows the search parameters to be refined. On the results page, the table of matching calibrators (Fig. 3) is listed. For all matching calibrators, the visibility and observability information is calculated and displayed. It is then possible to use VisCalc for a more comprehensive calculation of the visibility information.

Both tools can be accessed from the VLT Exposure Time Calculators page at www.eso.org/observing/etc. The standard version shows only those configurations that are offered for the current Call for Proposals. It is updated for each new Call for Proposals in order to reflect the offered VLTI baseline configurations and instrument modes. An expert version, accessible from the ETC preview page (www.eso.org/observing/etc/preview.html) offers an extended interface with many more choices. It supports the modes and configurations that are currently not offered. 


\section{Fourier Transform of Target}

\section{UV Plane ( uv tracks overlaid )}

Note: The start of each uv track is colored.

The shape and visibilities of this image below is dependent on the central wavelength used The UV coverage is $-200 \mathrm{~m}$ to $200 \mathrm{~m}$.

A baseline of $200 \mathrm{~m}$ at a wavelength of $10340.788 \mathrm{~nm}$ is equivalent to 0.094 cycles/mas

UV plane (showing MultiWavelength UvTracks)

Zoom FFT image (uv tracks removed, visibilities rescaled $0->100$ )

Fits file (uv tracks removed)

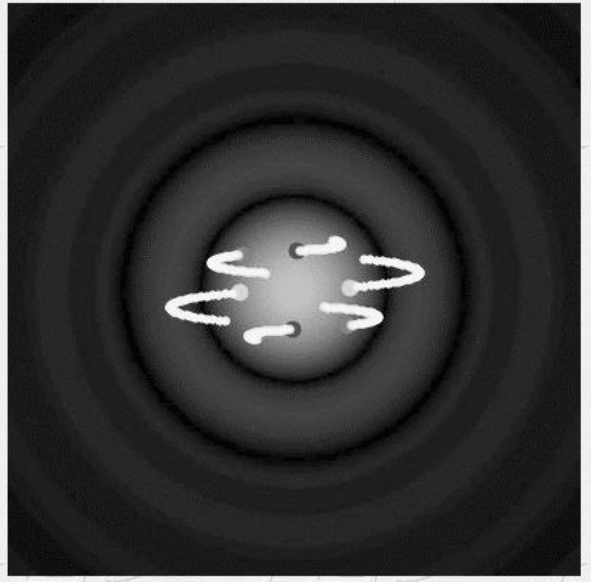

Visibility Squared (of uv points)

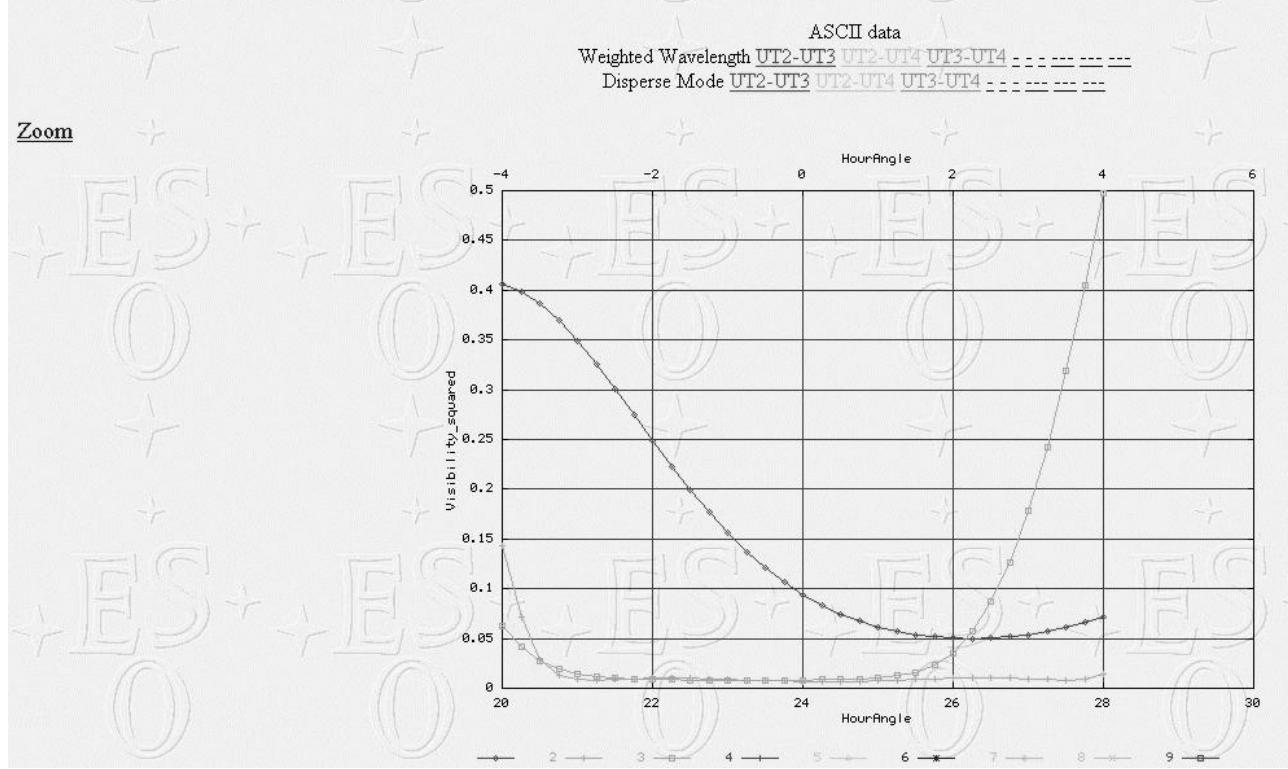

Figure 2. Sample output of the 2-d visibility function for MIDI with the $u v$-tracks overlayed (top) and the squared visibility as a function of hour angle (bottom) from the Visibility Calculator VisCalc. Chosen was a star at Dec $+7^{\circ}$ with uniform disk diameter of 40 mas and three P74 UT baselines. These outputs show the result for the central wavelength only; the information on the dispersed visibility values can be obtained as well. 


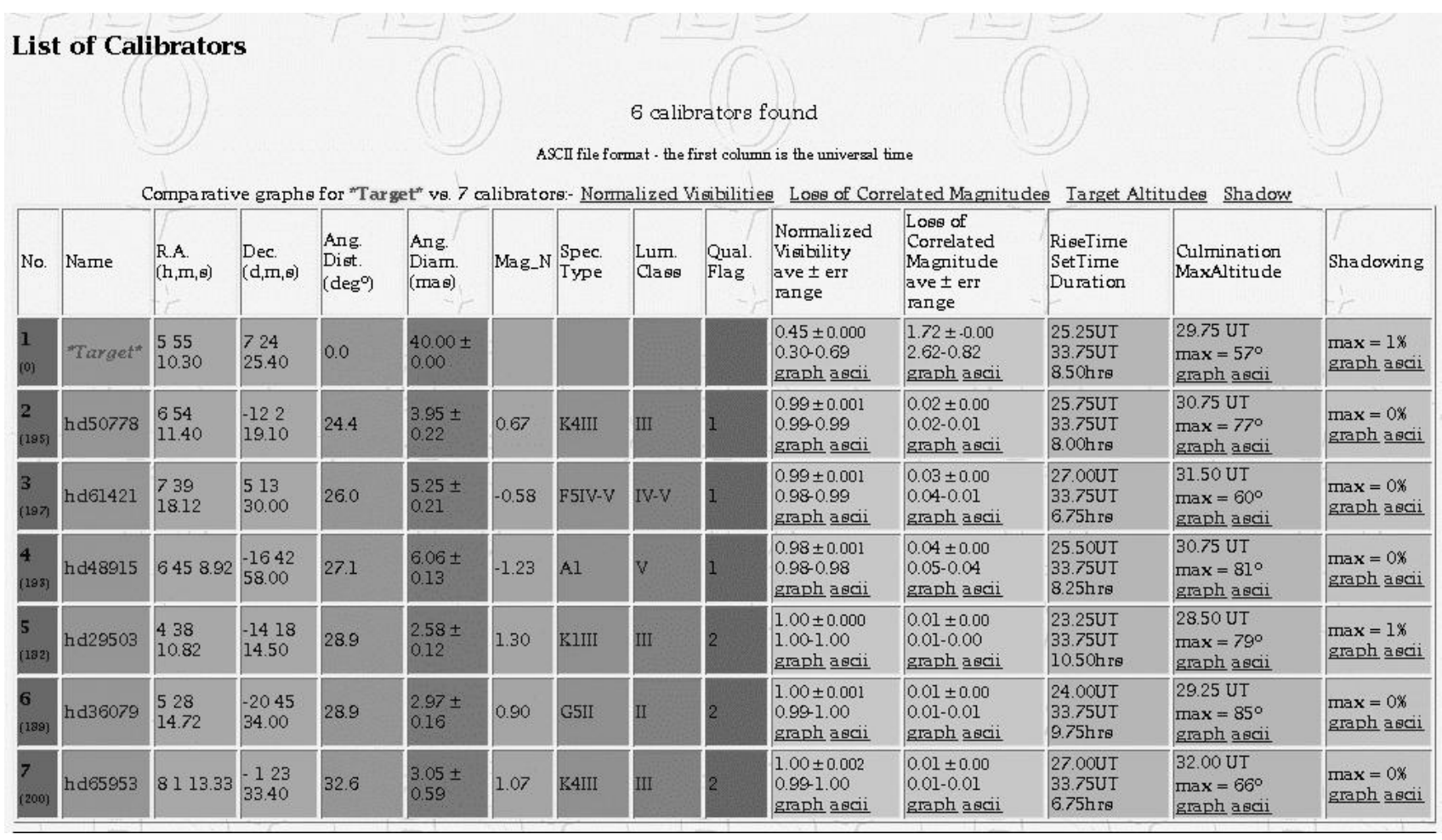

Figure 3. Sample output of the calibrator selection tool CalVin. Requested was a calibrator for Betelgeuse, with an angular distance of less than $35^{\circ}$, a diameter between 0 and 8 mas, and a magnitude between 1.3 and -5 .

\section{VLTI OBSERVING PERIODS - PAST, PRESENT, AND FUTURE}

Shared risk observations with the near-infrared $K$-band commissioning instrument VINCI in P70 and P71 During the ESO observing periods P70 and P71 in 2002, a total of 300h of shared-risk science observations were executed with the near-infrared $K$-band commissioning instrument VINCI and the $40 \mathrm{~cm}$ diameter VLTI test siderostats. While these operations did not yet follow exactly the VLT operations scheme, they helped greatly for the preparation of regular VLTI science operations, and tests of the complete data flow system. Several scientific results emerged from these observations, see Richichi \& Paresce $^{16}$ for a review. All these data, and also all data from commissioning using the VINCI instrument are publicly available at www.eso.org/projects/vlti/instru/vinci/vinci_data_sets.html.

MIDI science demonstration program. MIDI Science Demonstration activities of the VLTI with the Unit Telescopes have been ongoing since June 2003. A number of astronomical targets from various object classes have been observed in the framework of the Science Demonstration Time. These data are publicly available from the ESO archive (www.eso.org/projects/vlti/instru/midi/midi_data_sets.html). In addition to a scientific analysis, this programme allows the community as well to have a look at the data that can be obtained with MIDI. It allowed as well a first test of the data flow system for MIDI.

MIDI observations in P73. For the observing period P73, for observations between April 2004 and October 2004, VLTI observations with the MIDI instruments and two UT baselines were offered for the first time in service mode and visitor mode. One MIDI observing mode was offered, the dispersed-fringe mode, which uses a prism to provide a spectral resolution of $\mathrm{R} \sim 30$ at N-band (10 microns). A total of 30 programmes were proposed, out of which 20 programmes have been scheduled after the OPC rating, 12 for service mode, and 8 for visitor mode. These scheduled P73 programmes cover the scientific categories "B: Galactic Nuclei", "C: Interstellar medium, star and planet formation" and "D: Stellar evolution". The first block of P73 service mode observations using 
the UT2-UT3 baseline took place at the beginning of April 2004. A total of 30 pairs of science and calibration star OBs (30 hours of VLTI observing time, i.e. 60 hours of telescope time) have been successfully executed. Four service mode runs could be completed during this first observing period. The quality of these data is being assessed in Garching.

MIDI observations in P74. For observing period P74 (October 2004 to March 2005) VLTI observations with the MIDI instrument and three UT baselines were offered. This time, two modes can be used, the PRISM mode (spectral resolution of $R \sim 30$ ) and the GRISM mode (spectral resolution of $R \sim 230$ ), see Table 4. Again, 30 programmes, covering scientific categories $\mathrm{B}, \mathrm{C}$, and $\mathrm{D}$ (see above) were proposed. At the time of writing, the scheduling of these runs is not yet completed.

Upcoming observing periods While for the next observing period, P75 (April to October 2005), not many changes are expected, the following observing periods will be characterized by the addition of several new instruments and instrument modes. Over the next year, the near-infrared instrument AMBER, the external fringe tracker FINITO and the ATs will go into operations. AMBER currently undergoes its first commissioning run and it is likely that it will be offered for P76 (observations from October 2005). The first AT is on Paranal, the second will follow in the second half of 2004. If the commissioning goes ahead as planned, the first two ATs will be interferometrically combined in early 2005 and then they could also be included into the P76 call. The external fringe tracking facility FINITO is also in commissioning, which probably will be finalized only after the first ATs are available.

The addition of these new instruments and instrument modes will also require further development of the VLTI operations scheme in all phases from proposal preparation to data delivery. Especially with the additions of the ATs which can in principle be re-located over 30 possible stations, and the 3 -way beam combination with AMBER, further development of the available procedures and tools described in this article will become mandatory. In addition, while the current VLTI operations scheme as described so far has been proved to work, many details can be improved during the next observing periods.

\section{REFERENCES}

1. J. Alves \& J. Breysacher, "Telescope time allocation at ESO: the past, the present, and the future", Proc. SPIE 5493-28, in press

2. P. Ballester, T. Licha, D.J. McKay, S. Morel, I. Percheron, M. Petr-Gotzens, A. Richichi, C. Sabet, M. van den Ancker, M. Wittkowski, "The MIDI Data Flow: First Observing Period", The Messenger 116, in press, 2004

3. P. Ballester, et al., "Data flow system for the VLT interferometer", Proc. SPIE 5493-04, in press

4. F. Comerón, M. Romaniello, J. Breysacher, D. Silva, G. Mathys, The Messenger 113, pp. 32-36, 2003

5. E. Galliano, et al., "Selection of an optimum subset of telescope configurations for the VLTI", Proc. SPIE 5491-178, this volume

6. A. Glindemann et al., "VLTI technical advances (present and future)", Proc. SPIE 5491-50, this volume

7. C. Leinert, "Scientific observations with MIDI on the VLTI: present and future", Proc. SPIE 5491-03, this volume

8. D. J. McKay et al., "Observation planning tools for the ESO VLT interferometer", Proc. SPIE 5493-63, in press

9. Kimeswenger et al., "J-K DENIS photometry of a VLTI-selected sample of bright southern stars", A\&A 413, pp. 1037-1043, 2004

10. F. Malbet, et al., "Science program of the AMBER consortium", Proc. SPIE 5491-202, this volume

11. S. Morel, et al., "Preparing MIDI science operations at VLTI", Proc. SPIE 5491-193, this volume

12. I. Percheron, A. Richichi, M. Wittkowski, "Getting ready for high accuracy measurements: The VLTI Calibrators program", Proc. SPIE 4838, pp. 1424-1432, 2003

13. I. Percheron, A. Richichi, M. Wittkowski, "The VLTI Calibrators Program: Getting ready for high accuracy measurements", Ap\&SS 286, pp. 105-112, 2003 
14. I. Percheron, et al., "Quality Control and instrument trending for MIDI", Proc. SPIE 5491-134, this volume 15. F. T. Rantakyrö, et al., "VLTI science operations", Proc. SPIE 5491-196, this volume

16. A. Richichi, F. Paresce, "Harvesting Scientific Results with the VLTI", The Messenger 114, pp. 26-34, 2003

17. D. Silva, "Service mode scheduling: a primer for users", The Messenger 105, pp. 18-24, 2001 